\title{
Unfolding of eigenvalue surfaces near a diabolic point due to a complex perturbation
}

\author{
O. N. Kirillov, A. A. Mailybaev, and A. P. Seyranian \\ Institute of Mechanics, Moscow State Lomonosov University, \\ Michurinskii pr. 1, 119192 Moscow, Russia \\ E-mail: kirillov@imec.msu.ru
}

\begin{abstract}
The paper presents a new theory of unfolding of eigenvalue surfaces of real symmetric and Hermitian matrices due to an arbitrary complex perturbation near a diabolic point. General asymptotic formulae describing deformations of a conical surface for different kinds of perturbing matrices are derived. As a physical application, singularities of the surfaces of refractive indices in crystal optics are studied.
\end{abstract}

\section{Introduction}

Since the papers by Von Neumann and Wigner (1929) and Teller (1937) it is known that the energy surfaces in quantum physics may cross forming two sheets of a double cone: a diabolo. The apex of the cone is called a diabolic point, see Berry and Wilkinson (1984). This kind of crossing is typical for systems described by real symmetric Hamiltonians with at least two parameters and Hermitian Hamiltonians depending on three or more parameters. From mathematical point of view the energy surfaces are described by eigenvalues of real symmetric or Hermitian operators dependent on parameters, and the diabolic point is a point of a double eigenvalue with two linearly independent eigenvectors. In modern problems of quantum physics, crystal optics, 
physical chemistry, acoustics and mechanics it is important to know how the diabolic point bifurcates under arbitrary complex perturbations forming topological singularities of eigenvalue surfaces like a double coffee filter with two exceptional points or a diabolic circle of exceptional points, see e.g. Mondragon and Hernandez (1993), Mondragon and Hernandez (1996), Shuvalov and Scott (2000), Keck et al. (2003), Berry and Dennis (2003), Korsch and Mossman (2003), Berry (2004).

In our preceding companion paper Mailybaev et al. (2004), a general theory of coupling of eigenvalues for complex matrices of arbitrary dimension smoothly depending on multiple real parameters was presented. Two kinds of important singularities were mathematically classified: the diabolic points (DPs) and the exceptional points (EPs). DP is a point where the eigenvalues coalesce, while corresponding eigenvectors remain different (linearly independent), and EP is a point where both eigenvalues and eigenvectors coalesce forming a Jordan block. General formulae describing coupling and decoupling of eigenvalues, crossing and avoided crossing of eigenvalue surfaces were derived. Both the DP and EP cases are interesting in applications and were observed in experiments, see Ramachandran and Ramaseshan (1961), Dembowsky et al. (2001), Dembowsky et al. (2003), Stehmann et al. (2004).

In the present paper following the theory developed in Mailybaev et al. (2004) we study effects of complex perturbations in multiparameter families of real symmetric and Hermitian matrices. In case of real symmetric matrices we study unfolding of eigenvalue surfaces near a diabolic point under real and complex perturbations. Origination of singularities like a "double coffee filter" and a "diabolic circle" is analytically described. Unfolding of a diabolic point of a Hermitian matrix under an arbitrary complex perturbation is analytically treated. We emphasize that the unfolding of eigenvalue surfaces is described qualitatively as well as quantitatively by using only the information at the diabolic point, including eigenvalues, eigenvectors, and derivatives of the system matrix taken at the diabolic point.

As a physical application, singularities of the surfaces of refractive indices in crystal optics are studied. Asymptotic formulae for the metamorphoses of these surfaces depending on properties of a crystal are established and discussed in detail. Singular axes for general crystals with weak absorption and chirality are found. A new explicit condition distinguishing the absorbtion-dominated and chirality-dominated crystals is established in terms of components of the inverse dielectric tensor. 


\section{Asymptotic expressions for eigenvalues near a diabolic point}

Let us consider the eigenvalue problem

$$
\mathbf{A u}=\lambda \mathbf{u}
$$

for an $m \times m$ Hermitian matrix $\mathbf{A}$, where $\lambda$ is an eigenvalue and $\mathbf{u}$ is an eigenvector. Such eigenvalue problems arise in non-dissipative physics with and without time reversal symmetry. Real symmetric and complex Hermitian matrices correspond to these two cases, respectively. We assume that the matrix A smoothly depends on a vector of $n$ real parameters $\mathbf{p}=\left(p_{1}, \ldots, p_{n}\right)$. Let $\lambda_{0}$ be a double eigenvalue of the matrix $\mathbf{A}_{0}=\mathbf{A}\left(\mathbf{p}_{0}\right)$ for some vector $\mathbf{p}_{0}$. Since $\mathbf{A}_{0}$ is a Hermitian matrix, the eigenvalue $\lambda_{0}$ is real and possesses two eigenvectors $\mathbf{u}_{1}$ and $\mathbf{u}_{2}$. Thus, the point of eigenvalue coupling for Hermitian matrices is diabolic. We choose the eigenvectors satisfying the normalization conditions

$$
\left(\mathbf{u}_{1}, \mathbf{u}_{1}\right)=\left(\mathbf{u}_{2}, \mathbf{u}_{2}\right)=1, \quad\left(\mathbf{u}_{1}, \mathbf{u}_{2}\right)=0
$$

where the standard inner product of complex vectors is given by $(\mathbf{u}, \mathbf{v})=\sum_{i=1}^{m} u_{i} \bar{v}_{i}$.

Under perturbation of parameters $\mathbf{p}=\mathbf{p}_{0}+\Delta \mathbf{p}$, the bifurcation of $\lambda_{0}$ into two simple eigenvalues $\lambda_{+}$and $\lambda_{-}$occurs. The asymptotic formula for $\lambda_{ \pm}$under multiparameter perturbation is Mailybaev et al. (2004)

$$
\lambda_{ \pm}=\lambda_{0}+\frac{\left\langle\mathbf{f}_{11}+\mathbf{f}_{22}, \Delta \mathbf{p}\right\rangle}{2} \pm \sqrt{\frac{\left\langle\mathbf{f}_{11}-\mathbf{f}_{22}, \Delta \mathbf{p}\right\rangle^{2}}{4}+\left\langle\mathbf{f}_{12}, \Delta \mathbf{p}\right\rangle\left\langle\mathbf{f}_{21}, \Delta \mathbf{p}\right\rangle}
$$

Components of the vector $\mathbf{f}_{i j}=\left(f_{i j}^{1}, \ldots, f_{i j}^{n}\right)$ are

$$
f_{i j}^{k}=\left(\frac{\partial \mathbf{A}}{\partial p_{k}} \mathbf{u}_{i}, \mathbf{u}_{j}\right)
$$

where the derivative is taken at $\mathbf{p}_{0}$, and inner products of vectors in (3) are given by $\langle\mathbf{a}, \mathbf{b}\rangle=\sum_{i=1}^{n} a_{i} \bar{b}_{i}$. In expression (3) the higher order terms $o(\|\Delta \mathbf{p}\|)$ and $o\left(\|\Delta \mathbf{p}\|^{2}\right)$ are neglected before and under the square root. Since the matrix A is Hermitian, the vectors $\mathbf{f}_{11}$ and $\mathbf{f}_{22}$ are real and the vectors $\mathbf{f}_{12}=\overline{\mathbf{f}}_{21}$ are complex conjugate. In case of real symmetric matrices $\mathbf{A}=\mathbf{A}^{T}$, the vectors $\mathbf{f}_{11}, \mathbf{f}_{22}$, and $\mathbf{f}_{12}=\mathbf{f}_{21}$ are real. 
The asymptotic expression for the eigenvectors corresponding to $\lambda_{ \pm}$takes the form Mailybaev et al. (2004)

$$
\mathbf{u}_{ \pm}=\alpha_{ \pm} \mathbf{u}_{1}+\beta_{ \pm} \mathbf{u}_{2}, \quad \frac{\alpha_{ \pm}}{\beta_{ \pm}}=\frac{\left\langle\mathbf{f}_{12}, \Delta \mathbf{p}\right\rangle}{\lambda_{ \pm}-\lambda_{0}-\left\langle\mathbf{f}_{11}, \Delta \mathbf{p}\right\rangle}=\frac{\lambda_{ \pm}-\lambda_{0}-\left\langle\mathbf{f}_{22}, \Delta \mathbf{p}\right\rangle}{\left\langle\mathbf{f}_{21}, \Delta \mathbf{p}\right\rangle} .
$$

Expressions (5) provide zero order terms for the eigenvectors $\mathbf{u}_{ \pm}$under perturbation of the parameter vector.

Now, consider an arbitrary complex perturbation of the matrix family $\mathbf{A}(\mathbf{p})+$ $\Delta \mathbf{A}(\mathbf{p})$. Such perturbations appear due to non-conservative effects breaking symmetry of the initial system. We assume that the size of perturbation $\Delta \mathbf{A}(\mathbf{p}) \sim \varepsilon$ is small, where $\varepsilon=\left\|\Delta \mathbf{A}\left(\mathbf{p}_{0}\right)\right\|$ is the Frobenius norm of the perturbation at the diabolic point. Behavior of the eigenvalues $\lambda_{ \pm}$for small $\Delta \mathbf{p}$ and small $\varepsilon$ is described by the following asymptotic formula Mailybaev et al. (2004)

$$
\begin{aligned}
\lambda_{ \pm}= & \lambda_{0}+\frac{\left\langle\mathbf{f}_{11}+\mathbf{f}_{22}, \Delta \mathbf{p}\right\rangle}{2}+\frac{\varepsilon_{11}+\varepsilon_{22}}{2} \\
& \pm \sqrt{\frac{\left(\left\langle\mathbf{f}_{11}-\mathbf{f}_{22}, \Delta \mathbf{p}\right\rangle+\varepsilon_{11}-\varepsilon_{22}\right)^{2}}{4}+\left(\left\langle\mathbf{f}_{12}, \Delta \mathbf{p}\right\rangle+\varepsilon_{12}\right)\left(\left\langle\mathbf{f}_{21}, \Delta \mathbf{p}\right\rangle+\varepsilon_{21}\right) .}
\end{aligned}
$$

The quantities $\varepsilon_{i j}$ are small complex numbers of order $\varepsilon$ given by the expression

$$
\varepsilon_{i j}=\left(\Delta \mathbf{A}\left(\mathbf{p}_{0}\right) \mathbf{u}_{i}, \mathbf{u}_{j}\right) .
$$

A small variation of the matrix family leads to the following correction of the asymptotic expression for the eigenvectors

$$
\mathbf{u}_{ \pm}=\alpha_{ \pm}^{\varepsilon} \mathbf{u}_{1}+\beta_{ \pm}^{\varepsilon} \mathbf{u}_{2}, \quad \frac{\alpha_{ \pm}^{\varepsilon}}{\beta_{ \pm}^{\varepsilon}}=\frac{\left\langle\mathbf{f}_{12}, \Delta \mathbf{p}\right\rangle+\varepsilon_{12}}{\lambda_{ \pm}-\lambda_{0}-\left\langle\mathbf{f}_{11}, \Delta \mathbf{p}\right\rangle-\varepsilon_{11}}=\frac{\lambda_{ \pm}-\lambda_{0}-\left\langle\mathbf{f}_{22}, \Delta \mathbf{p}\right\rangle-\varepsilon_{22}}{\left\langle\mathbf{f}_{21}, \Delta \mathbf{p}\right\rangle+\varepsilon_{21}} .
$$

The ratios $\alpha_{+}^{\varepsilon} / \beta_{+}^{\varepsilon}=\alpha_{-}^{\varepsilon} / \beta_{-}^{\varepsilon}$ at the point of coincident eigenvalues $\lambda_{+}=\lambda_{-}$. Hence, the eigenvectors $\mathbf{u}_{+}=\mathbf{u}_{-}$coincide, and the point of eigenvalue coupling of the perturbed system becomes exceptional (EP). For some specific perturbations $\Delta \mathbf{A}(\mathbf{p})$, the coupling point may remain diabolic under the conditions

$$
\left\langle\mathbf{f}_{12}, \Delta \mathbf{p}\right\rangle+\varepsilon_{12}=0, \quad\left\langle\mathbf{f}_{21}, \Delta \mathbf{p}\right\rangle+\varepsilon_{21}=0, \quad\left\langle\mathbf{f}_{11}-\mathbf{f}_{22}, \Delta \mathbf{p}\right\rangle+\varepsilon_{11}-\varepsilon_{22}=0,
$$

when both ratios in (8) become undetermined.

We observe that asymptotic description of unfolding of diabolic singularity due to perturbation of the matrix family requires only the value of $\Delta \mathbf{A}(\mathbf{p})$ taken at the coupling point $\mathbf{p}_{0}$. Dependence of the perturbation $\Delta \mathrm{A}$ on the vector of parameters $\mathbf{p}$ near the point $\mathbf{p}_{0}$ is not so important, since it influences higher order terms. 


\section{Unfolding of a diabolic singularity for real sym- metric matrices}

Let us assume that $\mathbf{A}(\mathbf{p})$ is an $n$-parameter family of real symmetric matrices. Then its eigenvalues $\lambda$ are real. Let $\lambda_{0}$ be a double eigenvalue of the matrix $\mathbf{A}_{0}=\mathbf{A}\left(\mathbf{p}_{0}\right)$ with two real eigenvectors $\mathbf{u}_{1}$ and $\mathbf{u}_{2}$. Under perturbation of parameters $\mathbf{p}=\mathbf{p}_{0}+\Delta \mathbf{p}$, the eigenvalue $\lambda_{0}$ splits into two simple eigenvalues $\lambda_{+}$and $\lambda_{-}$. The asymptotic formula for $\lambda_{ \pm}$under multiparameter perturbation is given by equations (31) and (41), where the vectors $\mathbf{f}_{11}, \mathbf{f}_{22}$, and $\mathbf{f}_{12}=\mathbf{f}_{21}$ are real. Then, equation (3) takes the form

$$
\left(\lambda_{ \pm}-\lambda_{0}-\frac{\left\langle\mathbf{f}_{11}+\mathbf{f}_{22}, \Delta \mathbf{p}\right\rangle}{2}\right)^{2}-\frac{\left\langle\mathbf{f}_{11}-\mathbf{f}_{22}, \Delta \mathbf{p}\right\rangle^{2}}{4}-\left\langle\mathbf{f}_{12}, \Delta \mathbf{p}\right\rangle^{2}=0 .
$$

Equation (10) describes a surface in the space $\left(p_{1}, p_{2}, \ldots, p_{n}, \lambda\right)$, which consists of two sheets $\lambda_{+}(\mathbf{p})$ and $\lambda_{-}(\mathbf{p})$. These sheets are connected at the points satisfying the equations

$$
\lambda_{ \pm}=\lambda_{0}+\frac{1}{2}\left\langle\mathbf{f}_{11}+\mathbf{f}_{22}, \Delta \mathbf{p}\right\rangle, \quad\left\langle\mathbf{f}_{11}-\mathbf{f}_{22}, \Delta \mathbf{p}\right\rangle=0, \quad\left\langle\mathbf{f}_{12}, \Delta \mathbf{p}\right\rangle=0,
$$

where the eigenvalues couple: $\lambda_{+}=\lambda_{-}$. Equations (11) define a plane of dimension $n-2$. Thus, the double eigenvalue is a phenomenon of codimension 2 in an $n$ parameter family of real symmetric matrices Von Neumann and Wigner (1929).

For the two-parameter matrix $\mathbf{A}\left(p_{1}, p_{2}\right)$ equation (10) defines a double cone with apex at the point $\left(\mathbf{p}_{0}, \lambda_{0}\right)$ in the space $\left(p_{1}, p_{2}, \lambda\right)$, see Figure 1, The point $\left(\mathbf{p}_{0}, \lambda_{0}\right)$ is referred to as a "diabolic point" Berry and Wilkinson (1984) due to the conical shape of the children's toy "diabolo".

Let us consider a perturbation $\mathbf{A}(\mathbf{p})+\Delta \mathbf{A}(\mathbf{p})$ of the real symmetric family $\mathbf{A}(\mathbf{p})$ in the vicinity of the diabolic point $\mathbf{p}_{0}$, where $\Delta \mathbf{A}(\mathbf{p})$ is a complex matrix with the small norm $\varepsilon=\left\|\Delta \mathbf{A}\left(\mathbf{p}_{0}\right)\right\|$. Splitting of the double eigenvalue $\lambda_{0}$ due to a change of the vector of parameters $\Delta \mathbf{p}$ and a small complex perturbation $\Delta \mathrm{A}$ is described by equation (6), which acquires the form

$$
\lambda_{ \pm}=\lambda_{0}^{\prime}+\mu \pm \sqrt{c}, \quad c=(x+\xi)^{2}+(y+\eta)^{2}-\zeta^{2} .
$$

In equation (12) the quantities $\lambda_{0}^{\prime}, x$, and $y$ are real:

$$
\lambda_{0}^{\prime}=\lambda_{0}+\frac{1}{2}\left\langle\mathbf{f}_{11}+\mathbf{f}_{22}, \Delta \mathbf{p}\right\rangle, \quad x=\frac{1}{2}\left\langle\mathbf{f}_{11}-\mathbf{f}_{22}, \Delta \mathbf{p}\right\rangle, \quad y=\left\langle\mathbf{f}_{12}, \Delta \mathbf{p}\right\rangle,
$$




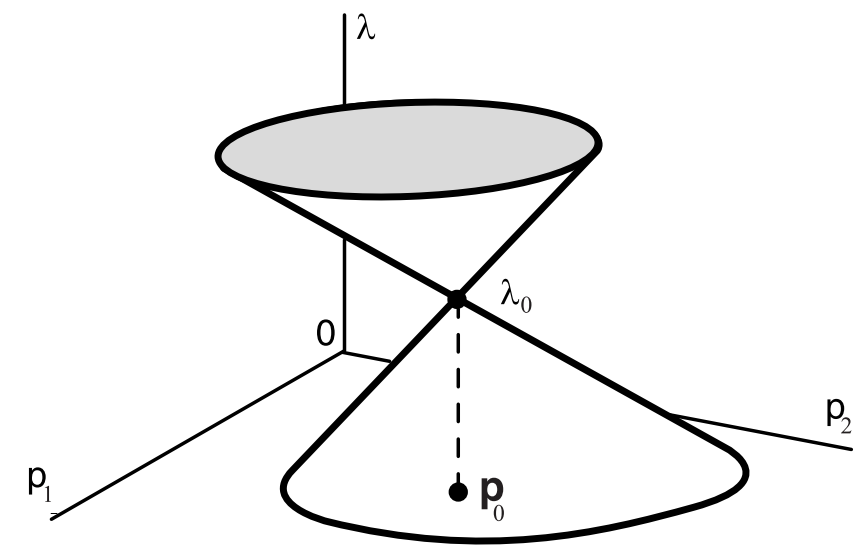

Figure 1: A diabolic point in a family of real symmetric matrices.

while the small coefficients $\mu, \xi, \eta$, and $\zeta$ are complex:

$$
\mu=\frac{1}{2}\left(\varepsilon_{11}+\varepsilon_{22}\right), \quad \xi=\frac{1}{2}\left(\varepsilon_{11}-\varepsilon_{22}\right), \quad \eta=\frac{1}{2}\left(\varepsilon_{12}+\varepsilon_{21}\right), \quad \zeta=\frac{1}{2}\left(\varepsilon_{12}-\varepsilon_{21}\right) .
$$

Separating real and imaginary parts in equation (12), we find

$$
\operatorname{Re}^{2}\left(\lambda-\lambda_{0}^{\prime}-\mu\right)-\operatorname{Im}^{2}\left(\lambda-\lambda_{0}^{\prime}-\mu\right)=\operatorname{Re} c, 2 \operatorname{Re}\left(\lambda-\lambda_{0}^{\prime}-\mu\right) \operatorname{Im}\left(\lambda-\lambda_{0}^{\prime}-\mu\right)=\operatorname{Im} c,
$$

where

$$
\begin{gathered}
\operatorname{Re} c=\left(\operatorname{Im}^{2} \zeta-\operatorname{Im}^{2} \xi-\operatorname{Im}^{2} \eta-\operatorname{Re}^{2} \zeta\right)+(x+\operatorname{Re} \xi)^{2}+(y+\operatorname{Re} \eta)^{2}, \\
\operatorname{Im} c=2((x+\operatorname{Re} \xi) \operatorname{Im} \xi+(y+\operatorname{Re} \eta) \operatorname{Im} \eta-\operatorname{Re} \zeta \operatorname{Im} \zeta) .
\end{gathered}
$$

From equations (15) we get the expressions determining the real and imaginary parts of the perturbed eigenvalues

$$
\begin{gathered}
\operatorname{Re} \lambda_{ \pm}=\lambda_{0}^{\prime}+\operatorname{Re} \mu \pm \sqrt{\left(\operatorname{Re} c+\sqrt{\operatorname{Re}^{2} c+\operatorname{Im}^{2} c}\right) / 2} \\
\operatorname{Im} \lambda_{ \pm}=\operatorname{Im} \mu \pm \sqrt{\left(-\operatorname{Re} c+\sqrt{\operatorname{Re}^{2} c+\operatorname{Im}^{2} c}\right) / 2}
\end{gathered}
$$

Strictly speaking, for the same eigenvalue one should take equal or opposite signs before the square roots in (18), (19) for positive or negative $\operatorname{Im} c$, respectively.

Equations (18) and (19) define surfaces in the spaces $\left(p_{1}, p_{2}, \ldots, p_{n}, \operatorname{Re} \lambda\right)$ and $\left(p_{1}, p_{2}, \ldots, p_{n}, \operatorname{Im} \lambda\right)$. Two sheets of the surface (18) are connected $\left(\operatorname{Re} \lambda_{+}=\operatorname{Re} \lambda_{-}\right)$at the points satisfying the conditions

$$
\operatorname{Re} c \leq 0, \quad \operatorname{Im} c=0, \quad \operatorname{Re} \lambda_{ \pm}=\lambda_{0}^{\prime}+\operatorname{Re} \mu
$$


while the sheets $\operatorname{Im} \lambda_{+}(\mathbf{p})$ and $\operatorname{Im} \lambda_{-}(\mathbf{p})$ are glued at the set of points satisfying

$$
\operatorname{Re} c \geq 0, \quad \operatorname{Im} c=0, \quad \operatorname{Im} \lambda_{ \pm}=\operatorname{Im} \mu .
$$

Note that in the neighborhood of the intersections (20) and (21) the eigenvalue sheets given by the formulae (18) and (19) can be described by the following approximate expressions

$$
\operatorname{Re} \lambda_{ \pm}=\lambda_{0}^{\prime}+\operatorname{Re} \mu \pm \frac{\operatorname{Im} c}{2} \sqrt{\frac{-1}{\operatorname{Re} c}}, \quad \operatorname{Re} c<0 ; \quad \operatorname{Im} \lambda_{ \pm}=\operatorname{Im} \mu \pm \frac{\operatorname{Im} c}{2} \sqrt{\frac{1}{\operatorname{Re} c}}, \quad \operatorname{Re} c>0 .
$$

The eigenvalue remains double under the perturbation of parameters when $c=0$, which yields two equations $\operatorname{Re} c=0$ and $\operatorname{Im} c=0$. Two cases are distinguished according to the sign of the quantity

$$
D=\operatorname{Im}^{2} \xi+\operatorname{Im}^{2} \eta-\operatorname{Im}^{2} \zeta
$$

If $D>0$, then the equations $\operatorname{Re} c=0$ and $\operatorname{Im} c=0$ with expressions (16), (17) yield two solutions $\left(x_{a}, y_{a}\right)$ and $\left(x_{b}, y_{b}\right)$, where

$$
\begin{aligned}
& x_{a, b}=\frac{\operatorname{Im} \xi \operatorname{Re} \zeta \operatorname{Im} \zeta \pm \operatorname{Im} \eta \sqrt{\left(\operatorname{Im}^{2} \xi+\operatorname{Im}^{2} \eta+\operatorname{Re}^{2} \zeta\right)\left(\operatorname{Im}^{2} \xi+\operatorname{Im}^{2} \eta-\operatorname{Im}^{2} \zeta\right)}}{\operatorname{Im}^{2} \xi+\operatorname{Im}^{2} \eta}-\operatorname{Re} \xi \\
& y_{a, b}=\frac{\operatorname{Im} \eta \operatorname{Re} \zeta \operatorname{Im} \zeta \mp \operatorname{Im} \xi \sqrt{\left(\operatorname{Im}^{2} \xi+\operatorname{Im}^{2} \eta+\operatorname{Re}^{2} \zeta\right)\left(\operatorname{Im}^{2} \xi+\operatorname{Im}^{2} \eta-\operatorname{Im}^{2} \zeta\right)}}{\operatorname{Im}^{2} \xi+\operatorname{Im}^{2} \eta}-\operatorname{Re} \eta .
\end{aligned}
$$

These two solutions determine the points in parameter space, where double eigenvalues appear. When $D=0$, the two solutions coincide. For $D<0$, the equations $\operatorname{Re} c=0$ and $\operatorname{Im} c=0$ have no real solutions. In the latter case, the eigenvalues $\lambda_{+}$and $\lambda_{-}$ separate for all $\Delta \mathbf{p}$.

Note that the quantities $\operatorname{Im} \xi$ and $\operatorname{Im} \eta$ are expressed by means of the anti-Hermitian part $\Delta \mathbf{A}_{N}=\left(\Delta \mathbf{A}-\overline{\Delta \mathbf{A}}^{T}\right) / 2$ of the matrix $\Delta \mathbf{A}$ as

$$
\begin{aligned}
& \operatorname{Im} \xi=\frac{\left(\Delta \mathbf{A}_{N}\left(\mathbf{p}_{0}\right) \mathbf{u}_{1}, \mathbf{u}_{1}\right)-\left(\Delta \mathbf{A}_{N}\left(\mathbf{p}_{0}\right) \mathbf{u}_{2}, \mathbf{u}_{2}\right)}{2 i} \\
& \operatorname{Im} \eta=\frac{\left(\Delta \mathbf{A}_{N}\left(\mathbf{p}_{0}\right) \mathbf{u}_{1}, \mathbf{u}_{2}\right)+\left(\Delta \mathbf{A}_{N}\left(\mathbf{p}_{0}\right) \mathbf{u}_{2}, \mathbf{u}_{1}\right)}{2 i}
\end{aligned}
$$

while $\operatorname{Im} \zeta$ depends on the Hermitian part $\Delta \mathbf{A}_{H}=\left(\Delta \mathbf{A}+\overline{\Delta \mathbf{A}}^{T}\right) / 2$ as

$$
\operatorname{Im} \zeta=\frac{\left(\Delta \mathbf{A}_{H}\left(\mathbf{p}_{0}\right) \mathbf{u}_{1}, \mathbf{u}_{2}\right)-\left(\Delta \mathbf{A}_{H}\left(\mathbf{p}_{0}\right) \mathbf{u}_{2}, \mathbf{u}_{1}\right)}{2 i}
$$



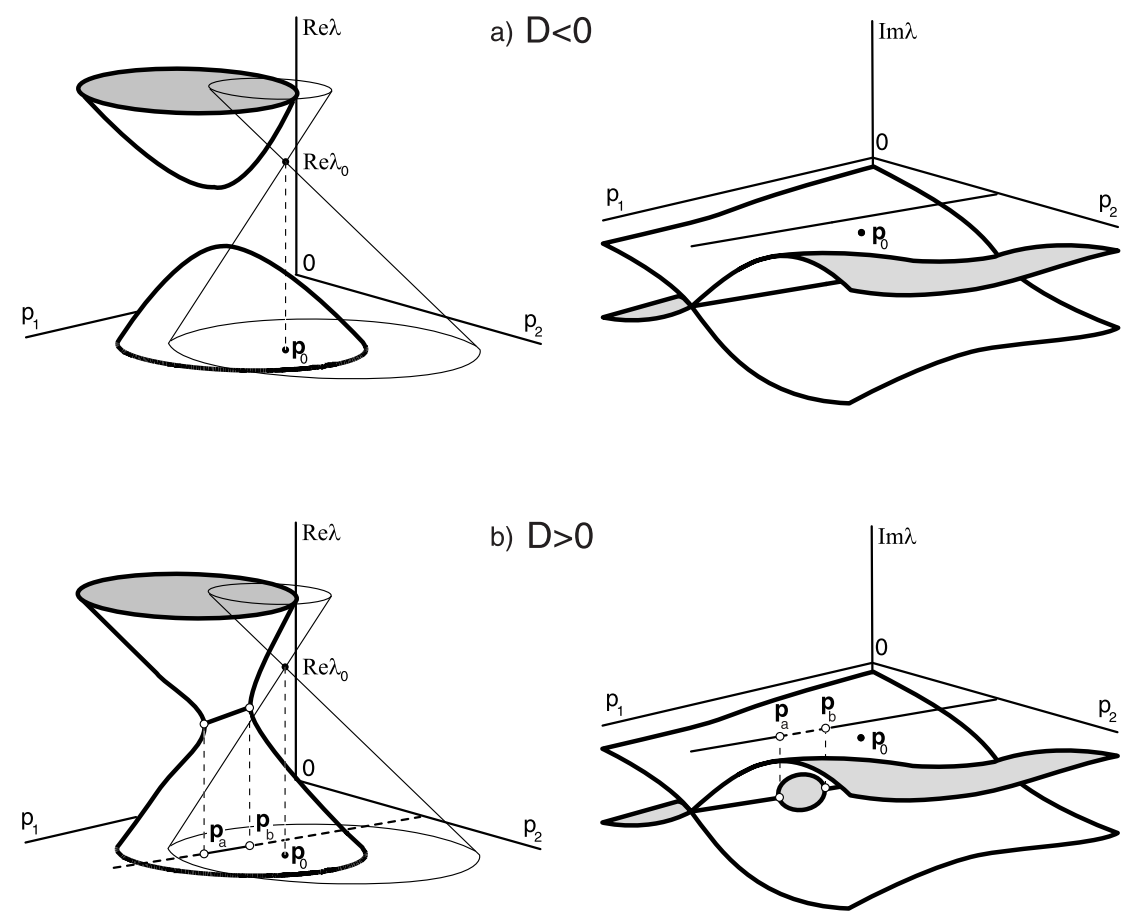

Figure 2: Unfolding of a diabolic point due to complex perturbation.

If $D>0$, one can say that the influence of the anti-Hermitian part of the perturbation $\Delta \mathbf{A}$ is stronger than that of the Hermitian part. If the Hermitian part prevails in the perturbation $\Delta \mathbf{A}$, we have $D<0$. In particular, $D=-\operatorname{Im}^{2} \zeta<0$ for a purely Hermitian perturbation $\Delta \mathbf{A}$.

Let us assume that the vector $\mathbf{p}$ consists of only two components $p_{1}$ and $p_{2}$, and consider the surfaces (18) and (19) for different kinds of the perturbation $\Delta \mathbf{A}(\mathbf{p})$. Consider first the case $D<0$. Then, the eigensheets $\operatorname{Re} \lambda_{+}(\mathbf{p})$ and $\operatorname{Re} \lambda_{-}(\mathbf{p})$ are separate, see Figure 2 . Indeed, for $D \leq-\operatorname{Re}^{2} \zeta$ the inequality $\operatorname{Rec} \geq 0$ holds for all variations of parameters, see equation (16). In the case when $-\operatorname{Re}^{2} \zeta<D<0$ the equation $\operatorname{Re} c=0$ with expressions (13) define an ellipse in the plane of parameters $\left(p_{1}, p_{2}\right)$. Inside the ellipse we have $\operatorname{Re} c<0$ and outside $\operatorname{Re} c>0$. Equation $\operatorname{Im} c=0$ defines a line in parameter plane. The line and the ellipse have no common points for $D<0$ since there are no real solutions of the equation $c=0$. Hence, for $D<0$ conditions (201) are not fulfilled and the real parts of the eigenvalues avoid crossing. As the size of the complex perturbation decreases $(\varepsilon \rightarrow 0)$, the two sheets come closer 
and touch each other at the point $\left(\mathbf{p}_{0}, \lambda_{0}\right)$ for $\varepsilon=0$ forming the diabolic singularity. The sheets $\operatorname{Im} \lambda_{+}(\mathbf{p})$ and $\operatorname{Im} \lambda_{-}(\mathbf{p})$ of the eigensurface (19) intersect along the line

$$
\operatorname{Im} c / 2=(x+\operatorname{Re} \xi) \operatorname{Im} \xi+(y+\operatorname{Re} \eta) \operatorname{Im} \eta-\operatorname{Re} \zeta \operatorname{Im} \zeta=0, \quad \operatorname{Im} \lambda_{ \pm}=\operatorname{Im} \mu,
$$

given by conditions (21). Note that, by using (22), one can show that the angle of intersection of the imaginary eigensheets is small of order $\varepsilon$ and tends to zero as $\varepsilon \rightarrow 0$.

In the case $D>0$ the line $\operatorname{Im} c=0$ and the ellipse $\operatorname{Re} c=0$ have common points $\mathbf{p}_{a}$ and $\mathbf{p}_{b}$ where the eigenvalues couple. Coordinates of these points found from the equations (13) are

$$
\mathbf{p}_{a, b}=\mathbf{p}_{0}+\left(-\frac{2 f_{12}^{2} x_{a, b}-\left(f_{11}^{2}-f_{22}^{2}\right) y_{a, b}}{f_{12}^{1}\left(f_{11}^{2}-f_{22}^{2}\right)-f_{12}^{2}\left(f_{11}^{1}-f_{22}^{1}\right)}, \quad \frac{2 f_{12}^{1} x_{a, b}-\left(f_{11}^{1}-f_{22}^{1}\right) y_{a, b}}{f_{12}^{1}\left(f_{11}^{2}-f_{22}^{2}\right)-f_{12}^{2}\left(f_{11}^{1}-f_{22}^{1}\right)}\right),
$$

where $x_{a, b}$ and $y_{a, b}$ are defined by expressions (24) and (25). Here we have assumed that the vectors $\mathbf{f}_{11}-\mathbf{f}_{22}$ and $\mathbf{f}_{12}$ are linearly independent. Note that the points $\mathbf{p}_{a}$ and $\mathbf{p}_{b}$ coincide in the degenerate case $D=0$.

According to conditions (20) the real eigensheets $\operatorname{Re} \lambda_{+}(\mathbf{p})$ and $\operatorname{Re} \lambda_{-}(\mathbf{p})$ are glued in the interval $\left[\mathbf{p}_{a}, \mathbf{p}_{b}\right]$ of the line

$$
\operatorname{Im} c / 2=(x+\operatorname{Re} \xi) \operatorname{Im} \xi+(y+\operatorname{Re} \eta) \operatorname{Im} \eta-\operatorname{Re} \zeta \operatorname{Im} \zeta=0, \quad \operatorname{Re} \lambda_{ \pm}=\lambda_{0}^{\prime}+\operatorname{Re} \mu
$$

The surface of real eigenvalues (18) is called a "double coffee filter" Keck et al. (2003). The unfolding of a diabolic point into the double coffee filter is shown in Figure $2 \mathrm{~b}$.

From conditions (21) it follows that the imaginary eigensheets $\operatorname{Im} \lambda_{+}(\mathbf{p})$ and $\operatorname{Im} \lambda_{-}(\mathbf{p})$ are connected along the straight line (28) where the interval $\left[\mathbf{p}_{a}, \mathbf{p}_{b}\right]$ is excluded, see Figure 2 $\mathrm{b}$. According to the formulae (22) the angle of intersection of the imaginary eigensheets tends to $\pi$ as the points $\mathbf{p}_{a}$ and $\mathbf{p}_{b}$ are approached, since Rec goes to zero. At far distances from the interval $\left[\mathbf{p}_{a}, \mathbf{p}_{b}\right]$ this angle becomes small of order $\varepsilon$. With the decrease of the size of complex perturbation $\varepsilon$ the interval shrinks and the angle of intersection goes to zero. At $\varepsilon=0$ the imaginary parts of the eigenvalues coincide: $\operatorname{Im} \lambda_{+}=\operatorname{Im} \lambda_{-}=0$. Note that in crystal optics and acoustics the interval $\left[\mathbf{p}_{a}, \mathbf{p}_{b}\right]$ is referred to as a "branch cut", and the points $\mathbf{p}_{a}, \mathbf{p}_{b}$ are called "singular axes", see Shuvalov and Scott (2000), Berry and Dennis (2003), Ramachandran and Ramaseshan (1961). According to equation (8) the double eigenvalues at $\mathbf{p}_{a}$ and $\mathbf{p}_{b}$ possess only one eigenvector and, hence, they are exceptional points (EPs). 

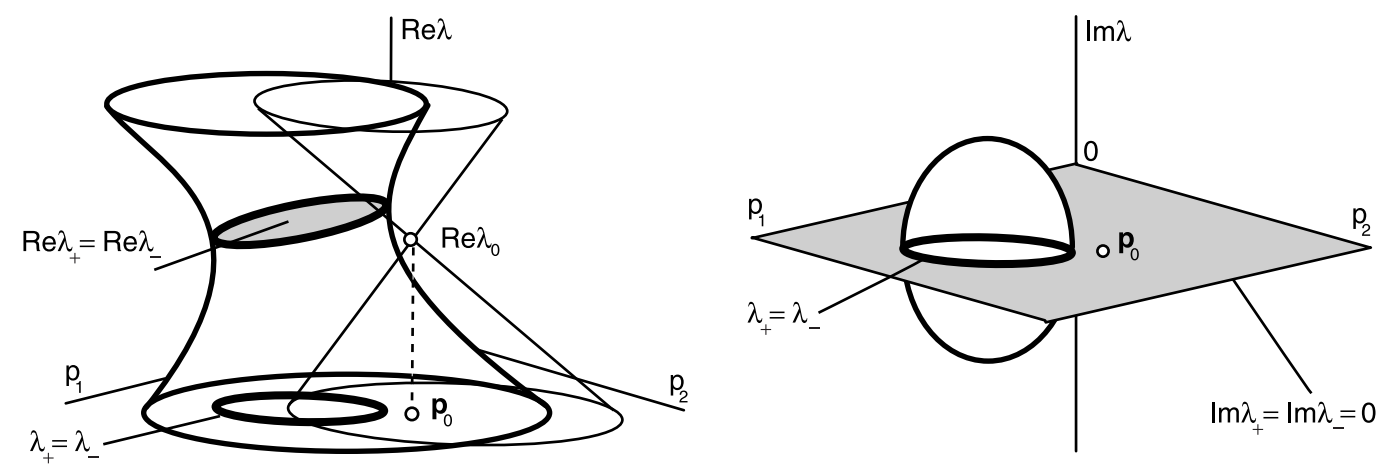

Figure 3: A real non-symmetric perturbation of a diabolic point.

Now let us consider the case when the perturbation $\Delta \mathbf{A}(\mathbf{p})$ is real. In this case $\mu$, $\xi, \eta, \zeta$, and hence,

$$
c=(x+\xi)^{2}+(y+\eta)^{2}-\zeta^{2}
$$

are real quantities. According to (12) the eigenvalues $\lambda_{ \pm}$are complex-conjugate if $c<0$ and real if $c>0$. The eigenvalues couple for $c=0$ forming a set consisting of exceptional points with double real eigenvalues.

Consider a system depending on a vector of two parameters $\mathbf{p}=\left(p_{1}, p_{2}\right)$. Then equation $c=0$ with expressions (13) and (31) define an ellipse in parameter plane; $c<0$ inside the ellipse and $c>0$ outside. Real parts of the eigenvalues are given by the equations

$$
\begin{array}{ll}
c \geq 0: & \left(\operatorname{Re} \lambda-\lambda_{0}^{\prime}-\mu\right)^{2}-(x+\xi)^{2}-(y+\eta)^{2}=-\zeta^{2}, \\
c \leq 0: & \operatorname{Re} \lambda=\lambda_{0}^{\prime}+\mu .
\end{array}
$$

Equation (32) defines a hyperboloid in the space $\left(p_{1}, p_{2}, \operatorname{Re} \lambda\right)$. Real parts of the eigenvalues $\lambda_{ \pm}$coincide at the disk determined by equation (33), see Figure 3. Imaginary parts of the eigenvalues are

$$
\begin{array}{ll}
c \geq 0: & \operatorname{Im} \lambda=0, \\
c \leq 0: & \operatorname{Im}^{2} \lambda+(x+\xi)^{2}+(y+\eta)^{2}=\zeta^{2} .
\end{array}
$$

The imaginary parts are both zero at the points of the plane (134) surrounding the ellipsoid (35) ("a bubble") in the space $\left(p_{1}, p_{2}, \operatorname{Im} \lambda\right)$, see Figure 3 . The eigenvalues couple at the points of the elliptic ring

$$
\lambda_{ \pm}=\lambda_{0}^{\prime}+\mu, \quad(x+\xi)^{2}+(y+\eta)^{2}=\zeta^{2},
$$


consisting of exceptional points, see Figure 3. By that reason we call it an "exceptional ring", which is a better name compared with a "diabolic circle" suggested by Mondragon and Hernandez (1993), Mondragon and Hernandez (1996).

Finally, it is instructive to consider deformations of the surfaces (132), (133) and (34), (35) as the real perturbation becomes complex. If the imaginary part of the perturbation $\operatorname{Im} \Delta \mathbf{A}$ is such that $D<0$, then the parts of the hyperboloid (32) connected by the disk (33) are separated into the two smooth surfaces described by the equation (18). On the other hand, the ellipsoid (35) surrounded by the plane (34) is foliated into two sheets crossing each other along the line $\operatorname{Im} c=0$, see Figure $2 \mathrm{a}$. Recall that the line $\operatorname{Im} c=0$ does not intersect the ellipse $\operatorname{Re} c=0$. When $D>0$, the disk (33) foliates into two sheets crossing along the interval $\left[\mathbf{p}_{a}, \mathbf{p}_{b}\right]$, where the points $\mathbf{p}_{a}$ and $\mathbf{p}_{b}$ are given by expression (29). As the size of the imaginary part of the perturbation $\operatorname{Im} \Delta \mathbf{A}$ increases, the angle of intersection of real eigensheets grows. By this way, the purely imaginary perturbation deforms the hyperboloid (32) into the double coffee filter (18), see Figure 2b. The ellipsoid (35) surrounded by the plane (134) is transformed into two smooth sheets intersecting along the line $\operatorname{Im} c=0$, where the interval $\left[\mathbf{p}_{a}, \mathbf{p}_{b}\right]$ is excluded. The angle of intersection grows as the size of the perturbation $\operatorname{Im} \Delta \mathbf{A}$ increases.

\section{Unfolding of a diabolic singularity for Hermitian matrices}

Let us consider a multi-parameter Hermitian matrix $\mathbf{A}(\mathbf{p})$. Assume that $\mathbf{p}_{0}$ is a diabolic point, where the matrix $\mathbf{A}_{0}=\mathbf{A}\left(\mathbf{p}_{0}\right)$ has a double real eigenvalue $\lambda_{0}$ with two eigenvectors. The splitting of $\lambda_{0}$ into a pair of simple real eigenvalues $\lambda_{+}$and $\lambda_{-}$is described by expressions (3), (4), where the vectors $\mathbf{f}_{11}$ and $\mathbf{f}_{22}$ are real and the vectors $\mathbf{f}_{12}=\overline{\mathbf{f}}_{21}$ are complex conjugate. By using expression (3), we find

$$
\lambda_{ \pm}=\lambda_{0}^{\prime} \pm \sqrt{x^{2}+y^{2}+z^{2}}
$$


where $\lambda_{0}^{\prime}, x, y$, and $z$ are real quantities depending linearly on the perturbation of parameters $\Delta \mathbf{p}$ as follows

$$
\lambda_{0}^{\prime}=\lambda_{0}+\frac{\left\langle\mathbf{f}_{11}+\mathbf{f}_{22}, \Delta \mathbf{p}\right\rangle}{2}, x=\frac{\left\langle\mathbf{f}_{11}-\mathbf{f}_{22}, \Delta \mathbf{p}\right\rangle}{2}, y=\left\langle\operatorname{Re} \mathbf{f}_{12}, \Delta \mathbf{p}\right\rangle, z=\left\langle\operatorname{Im} \mathbf{f}_{12}, \Delta \mathbf{p}\right\rangle .
$$

The eigenvalues coincide if $x=y=z=0$. Thus, if the system depends on three parameters and the real vectors $\mathbf{f}_{11}-\mathbf{f}_{22}, \operatorname{Re} \mathbf{f}_{12}$, and $\operatorname{Im} \mathbf{f}_{12}$ are linearly independent, the eigenvalues $\lambda_{+}$and $\lambda_{-}$split for any nonzero perturbation $\Delta \mathbf{p}$. For more than three parameters, the equations $x=y=z=0$ with relations (38) provide a plane of diabolic points in parameter space. This plane has dimension $n-3$, which agrees with the well-known fact that the diabolic point is a codimension 3 phenomenon for Hermitian systems Von Neumann and Wigner (1929), Arnold (1972).

Now let us consider a general non-Hermitian perturbation of the system $\mathbf{A}(\mathbf{p})+$ $\Delta \mathbf{A}(\mathbf{p})$, assuming that the size of perturbation at the diabolic point $\varepsilon=\left\|\Delta \mathbf{A}\left(\mathbf{p}_{0}\right)\right\|$ is small. The two eigenvalues $\lambda_{+}$and $\lambda_{-}$, which become complex due to non-Hermitian perturbation, are given by asymptotic expressions (6), (7). With the use of the new coordinates (38), we write the expression (6) as

$$
\lambda_{ \pm}=\lambda_{0}^{\prime}+\mu \pm \sqrt{c}
$$

where

$$
c=(x+\xi)^{2}+(y+\eta)^{2}+(z-i \zeta)^{2},
$$

and $\mu, \xi, \eta, \zeta$ are small complex quantities of order $\varepsilon$ given by expressions (14).

The eigenvalues couple $\left(\lambda_{+}=\lambda_{-}\right)$if $c=0$. This yields two equations

$$
\begin{gathered}
\operatorname{Re} c=(x+\operatorname{Re} \xi)^{2}+(y+\operatorname{Re} \eta)^{2}+(z+\operatorname{Im} \zeta)^{2}-\left(\operatorname{Im}^{2} \xi+\operatorname{Im}^{2} \eta+\operatorname{Re}^{2} \zeta\right)=0 \\
\operatorname{Im} c=2(\operatorname{Im} \xi(x+\operatorname{Re} \xi)+\operatorname{Im} \eta(y+\operatorname{Re} \eta)-\operatorname{Re} \zeta(z+\operatorname{Im} \zeta))=0 .
\end{gathered}
$$

Equation (41) defines a sphere in $(x, y, z)$ space with the center at $(-\operatorname{Re} \xi,-\operatorname{Re} \eta,-\operatorname{Im} \zeta)$ and the radius $\sqrt{\operatorname{Im}^{2} \xi+\operatorname{Im}^{2} \eta+\operatorname{Re}^{2} \zeta}$, which are small of order $\varepsilon$. Equation (42) yields a plane passing through the center of the sphere. The sphere and the plane intersect along a circle. Points of this circle determine values of parameters, for which the eigenvalues $\lambda_{ \pm}$coincide. Since $c=0$ at the coupling point, expression (8) for the eigenvectors takes the form

$$
\mathbf{u}_{ \pm}=\alpha_{ \pm}^{\varepsilon} \mathbf{u}_{1}+\beta_{ \pm}^{\varepsilon} \mathbf{u}_{2}, \quad \frac{\alpha_{ \pm}^{\varepsilon}}{\beta_{ \pm}^{\varepsilon}}=\frac{y+i z+\eta+\zeta}{-x-\xi}=\frac{x+\xi}{y-i z+\eta-\zeta}
$$




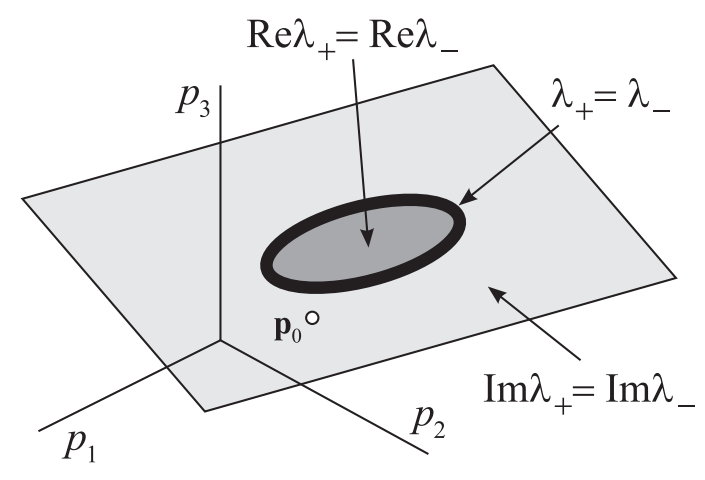

Figure 4: Unfolding of a diabolic point into an exceptional ring in parameter space.

Thus, all points of the circle are exceptional points, where the two eigenvectors $\mathbf{u}_{-}$ and $\mathbf{u}_{+}$merge in addition to the coupling of the eigenvalues $\lambda_{+}$and $\lambda_{-}$. By using the linear expressions (38), the set of exceptional points is found in the original parameter space p. The exceptional circle in $(x, y, z)$ space is transformed into an exceptional elliptic ring in three-parameter space $\mathbf{p}$, see Figure 4

Let us consider the plane (42), at which the quantity $c$ is real. By formula (39), the real parts of the eigenvalues $\lambda_{ \pm}$coincide inside the exceptional ring, where $c<0$, and the imaginary parts of $\lambda_{ \pm}$coincide outside the exceptional ring, where $c>0$, see the dark and light shaded areas in Figure 4.

We see that, under a general complex perturbation, a diabolic point of a threeparameter Hermitian system bifurcates into an exceptional ring. This ring has elliptic shape and grows proportionally to the size of perturbation $\varepsilon$. The real and imaginary parts of the eigenvalues $\lambda_{ \pm}$coincide, respectively, inside and outside the exceptional ring in the plane of the ring.

Finally, let us study the stratification of parameter space given by the condition $\left|\operatorname{Re}\left(\lambda_{+}-\lambda_{-}\right)\right|=$const. For problems of quantum mechanics, this difference describes the size of a gap between two adjacent energy levels. By using expression (39), we find $\left(\lambda_{+}-\lambda_{-}\right)^{2}=4 c$. Separating real and imaginary parts in this equation and extracting $\operatorname{Im}\left(\lambda_{+}-\lambda_{-}\right)$, we get

$$
\operatorname{Re}^{4}\left(\lambda_{+}-\lambda_{-}\right)-4 \operatorname{Re}^{2}\left(\lambda_{+}-\lambda_{-}\right) \operatorname{Re} c-4 \operatorname{Im}^{2} c=0,
$$

where $\operatorname{Re} c$ and $\operatorname{Im} c$ are given by the first equalities in (41) and (42). Given fixed value of $\left|\operatorname{Re}\left(\lambda_{+}-\lambda_{-}\right)\right|$, equation (44) with (41), (42), and (38) define an ellipsoid in 
(a)

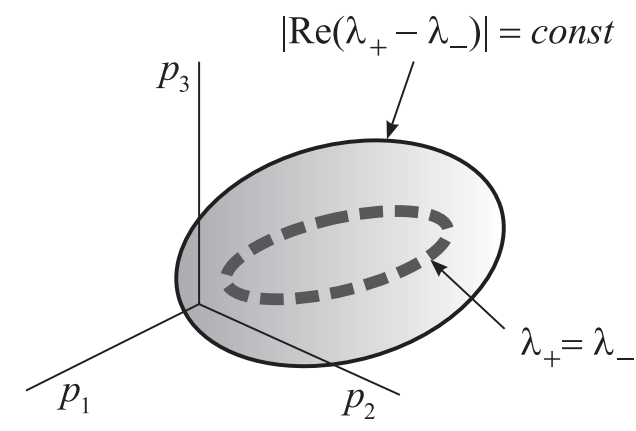

(b)

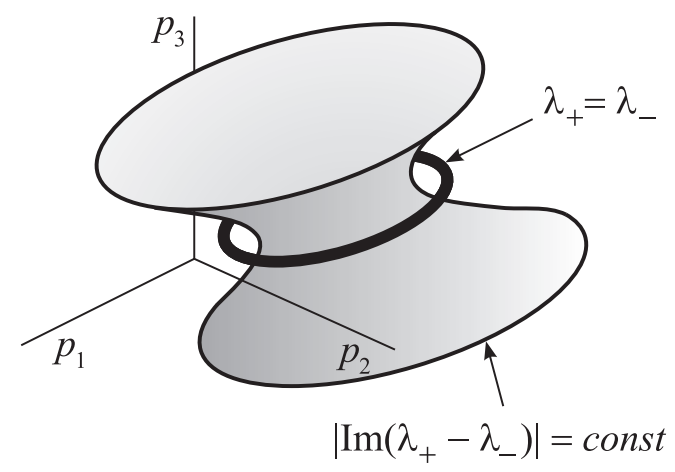

Figure 5: Surfaces corresponding to coincident real or imaginary parts of eigenvalues.

three-parameter space enclosing the exceptional ring, see Figure 5 a. Similar analysis provides the equation

$$
\operatorname{Im}^{4}\left(\lambda_{+}-\lambda_{-}\right)+4 \operatorname{Im}^{2}\left(\lambda_{+}-\lambda_{-}\right) \operatorname{Re} c-4 \operatorname{Im}^{2} c=0
$$

for a surface given by the condition $\left|\operatorname{Im}\left(\lambda_{+}-\lambda_{-}\right)\right|=$const. In three-parameter space equation (45) defines a hyperboloid surrounded by the exceptional ring, see Figure 5b.

\section{Unfolding of optical singularities of birefringent crystals}

Optical properties of a non-magnetic dichroic chiral anisotropic crystal are characterized by the inverse dielectric tensor $\boldsymbol{\eta}$, which relates the vectors of electric field $\mathbf{E}$ and the displacement D as Landau et al. (1984)

$$
\mathbf{E}=\eta \mathrm{D}
$$

A monochromatic plane wave of frequency $\omega$ that propagates in a direction specified by a real unit vector $\mathbf{s}=\left(s_{1}, s_{2}, s_{3}\right)$ has the form

$$
\mathbf{D}(\mathbf{r}, t)=\mathbf{D}(\mathbf{s}) \exp i \omega\left(\frac{n(\mathbf{s})}{c} \mathbf{s}^{T} \mathbf{r}-t\right),
$$


where $n(\mathbf{s})$ is a refractive index, and $\mathbf{r}$ is the real vector of spatial coordinates. With the wave (47) and the constitutive relation (46) Maxwell's equations after some elementary manipulations yield (see e.g. Berry and Dennis (2003) )

$$
\boldsymbol{\eta} \mathbf{D}(\mathbf{s})-\mathbf{s s}^{T} \boldsymbol{\eta} \mathbf{D}(\mathbf{s})=\frac{1}{n^{2}(\mathbf{s})} \mathbf{D}(\mathbf{s}) .
$$

Multiplying equation (48) by the vector $\mathbf{s}^{T}$ from the left, we find that for plane waves the vector $\mathbf{D}$ is always orthogonal to the direction $\mathbf{s}$, i.e., $\mathbf{s}^{T} \mathbf{D}(\mathbf{s})=0$. By using this condition, we write (48) in the form of the eigenvalue problem

$$
\left[\left(\mathbf{I}-\mathbf{s s}^{T}\right) \boldsymbol{\eta}\left(\mathbf{I}-\mathbf{s s}^{T}\right)\right] \mathbf{u}=\lambda \mathbf{u}
$$

where $\lambda=n^{-2}, \mathbf{u}=\mathbf{D}$, and $\mathbf{I}$ is the identity matrix. Since $\mathbf{I}-\mathbf{s s}^{T}$ is a singular matrix, one of the eigenvalues is always zero. Let us denote the other two eigenvalues by $\lambda_{+}$ and $\lambda_{-}$. These eigenvalues determine refractive indices $n$, and the corresponding eigenvectors yield polarizations.

The inverse dielectric tensor is described by a complex non-Hermitian matrix $\boldsymbol{\eta}=$ $\boldsymbol{\eta}_{\text {transp }}+\boldsymbol{\eta}_{\text {dichroic }}+\boldsymbol{\eta}_{\text {chiral }}$. The symmetric part of $\boldsymbol{\eta}$ consisting of the real matrix $\boldsymbol{\eta}_{\text {transp }}$ and imaginary matrix $\boldsymbol{\eta}_{\text {dichroic }}$ constitute the anisotropy tensor, which describes the birefringence of the crystal. For a transparent crystal, the anisotropy tensor is real and is represented only by the matrix $\boldsymbol{\eta}_{\text {transp }}$; for a crystal with linear dichroism it is complex. Choosing coordinate axes along the principal axes of $\boldsymbol{\eta}_{\text {transp }}$, we have

$$
\boldsymbol{\eta}_{\text {transp }}=\left(\begin{array}{ccc}
\eta_{1} & 0 & 0 \\
0 & \eta_{2} & 0 \\
0 & 0 & \eta_{3}
\end{array}\right)
$$

The matrix

$$
\boldsymbol{\eta}_{\text {dichroic }}=i\left(\begin{array}{ccc}
\eta_{11}^{d} & \eta_{12}^{d} & \eta_{13}^{d} \\
\eta_{12}^{d} & \eta_{22}^{d} & \eta_{23}^{d} \\
\eta_{13}^{d} & \eta_{23}^{d} & \eta_{33}^{d}
\end{array}\right)
$$

describes linear dichroism (absorption). The matrix $\boldsymbol{\eta}_{\text {chiral }}$ gives the antisymmetric part of $\boldsymbol{\eta}$ describing chirality (optical activity) of the crystal. It is determined by the optical activity vector $\mathbf{g}=\left(g_{1}, g_{2}, g_{3}\right)$ depending linearly on $\mathbf{s}$ as

$$
\boldsymbol{\eta}_{\text {chiral }}=i\left(\begin{array}{ccc}
0 & -g_{3} & g_{2} \\
g_{3} & 0 & -g_{1} \\
-g_{2} & g_{1} & 0
\end{array}\right), \quad \mathbf{g}=\boldsymbol{\gamma} \mathbf{s}=\left(\begin{array}{ccc}
\gamma_{11} & \gamma_{12} & \gamma_{13} \\
\gamma_{12} & \gamma_{22} & \gamma_{23} \\
\gamma_{13} & \gamma_{23} & \gamma_{33}
\end{array}\right)\left(\begin{array}{c}
s_{1} \\
s_{2} \\
s_{3}
\end{array}\right)
$$


where $\gamma$ is a symmetric optical activity tensor; this tensor has an imaginary part for a material with circular dichroism, see Berry and Dennis (2003) for more details.

First, consider a transparent non-chiral crystal, when $\boldsymbol{\eta}_{\text {dichroic }}=0$ and $\boldsymbol{\gamma}=0$. Then the matrix

$$
\mathbf{A}(\mathbf{p})=\left(\mathbf{I}-\mathbf{s s}^{T}\right) \boldsymbol{\eta}_{\text {transp }}\left(\mathbf{I}-\mathbf{s s}^{T}\right)
$$

is real symmetric and depends on a vector of two parameters $\mathbf{p}=\left(s_{1}, s_{2}\right)$ (see Berry and Dennis (2003) for other ways of introducing two parameters). The third component of the direction vector $\mathbf{s}$ is found as $s_{3}= \pm \sqrt{1-s_{1}^{2}-s_{2}^{2}}$, where the cases of two different signs should be considered separately. Below we assume that three dielectric constants $\eta_{1}>\eta_{2}>\eta_{3}$ are different. This corresponds to biaxial anisotropic crystals.

The nonzero eigenvalues $\lambda_{ \pm}$of the matrix $\mathbf{A}(\mathbf{p})$ are found explicitly in the form Lewin (1994)

$$
\lambda_{ \pm}=\frac{\operatorname{trace} \mathbf{A}}{2} \pm \frac{1}{2} \sqrt{2 \operatorname{trace}\left(\mathbf{A}^{2}\right)-(\operatorname{trace} \mathbf{A})^{2}} .
$$

The eigenvalues $\lambda_{ \pm}$are the same for opposite directions $\mathbf{s}$ and $\mathbf{-} \mathbf{s}$. By using (50) and (53) in (154), it is straightforward to show that two eigenvalues $\lambda_{+}$and $\lambda_{-}$couple at

$$
\mathbf{s}_{0}=\left(S_{1}, S_{2}, S_{3}\right), \lambda_{0}=\eta_{2} ; \quad S_{1}= \pm \sqrt{\left(\eta_{1}-\eta_{2}\right) /\left(\eta_{1}-\eta_{3}\right)}, S_{2}=0, S_{3}= \pm \sqrt{1-S_{1}^{2}}
$$

which determine four diabolic points (for two signs of $S_{1}$ and $S_{3}$ ), also called optic axes Ramachandran and Ramaseshan (1961). The double eigenvalue $\lambda_{0}=\eta_{2}$ of the matrix $\mathbf{A}_{0}=\mathbf{A}\left(\mathbf{p}_{0}\right), \mathbf{p}_{0}=\left(S_{1}, 0\right)$ possesses two eigenvectors

$$
\mathbf{u}_{1}=\left(\begin{array}{c}
0 \\
1 \\
0
\end{array}\right), \quad \mathbf{u}_{2}=\left(\begin{array}{c}
S_{3} \\
0 \\
-S_{1}
\end{array}\right)
$$

satisfying normalization conditions (21). Using expressions (53) and (156), we evaluate the vectors $\mathbf{f}_{i j}$ with components (44) for optic axes (55) as

$$
\mathbf{f}_{11}=(0,0), \quad \mathbf{f}_{22}=\left(2\left(\eta_{3}-\eta_{1}\right) S_{1}, 0\right), \quad \mathbf{f}_{12}=\mathbf{f}_{21}=\left(0,\left(\eta_{3}-\eta_{1}\right) S_{1} S_{3}\right)
$$

By using (55) and (57) in (10), we obtain the local asymptotic expression for the cone singularities in the space $\left(s_{1}, s_{2}, \lambda\right)$ as

$$
\left(\lambda-\eta_{2}-\left(\eta_{3}-\eta_{1}\right) S_{1}\left(s_{1}-S_{1}\right)\right)^{2}=\left(\eta_{3}-\eta_{1}\right)^{2} S_{1}^{2}\left(\left(s_{1}-S_{1}\right)^{2}+S_{3}^{2} s_{2}^{2}\right)
$$




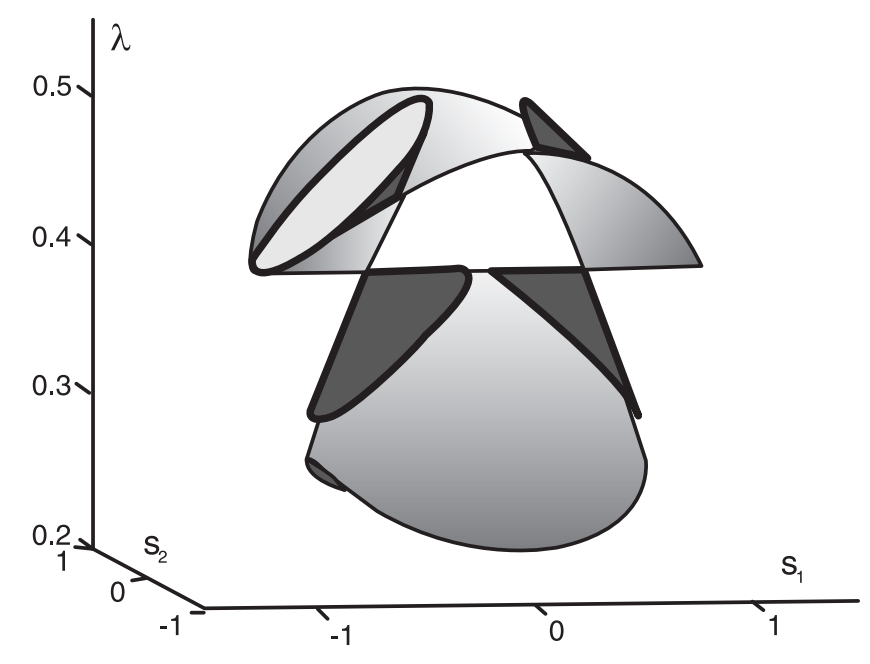

Figure 6: Diabolic singularities near optic axes and their local approximations.

Equation (58) is valid for each of the four optic axes (55).

As an example, consider the case of $\eta_{1}=0.5, \eta_{2}=0.4, \eta_{3}=0.1$. Conical surfaces (58) are shown in Figure 6 together with the exact eigenvalue surfaces (54). The two optic axes presented in Figure 6 are $\mathbf{s}_{0}=( \pm 1 / 2,0, \sqrt{3} / 2)$ with the double eigenvalue $\lambda_{0}=2 / 5 ;$ the eigenvalue surfaces for the opposite directions $\mathbf{s}_{0}=( \pm 1 / 2,0,-\sqrt{3} / 2)$ are exactly the same.

Now let us assume that the crystal possesses absorption and chirality. Then the matrix family (53) takes a complex perturbation $\mathbf{A}(\mathbf{p})+\Delta \mathbf{A}(\mathbf{p})$, where

$$
\Delta \mathbf{A}(\mathbf{p})=\left(\mathbf{I}-\mathbf{s s}^{T}\right)\left(\boldsymbol{\eta}_{\text {dichroic }}+\boldsymbol{\eta}_{\text {chiral }}\right)\left(\mathbf{I}-\mathbf{s s}^{T}\right) .
$$

Assume that the absorption and chirality are weak, i.e., $\varepsilon=\left\|\boldsymbol{\eta}_{\text {dichroic }}\right\|+\left\|\boldsymbol{\eta}_{\text {chiral }}\right\|$ is small. Then we can use asymptotic formulae of Sections 2 and 3 to describe unfolding of diabolic singularities of the eigenvalue surfaces. For this purpose, we need to know only the value of the perturbation $\Delta \mathbf{A}$ at the optic axes of the transparent non-chiral crystal $\mathbf{s}_{0}$.

Substituting matrix (59) evaluated at optic axes (55) into expression (77), we obtain

$$
\begin{gathered}
\varepsilon_{11}=i \eta_{22}^{d}, \quad \varepsilon_{22}=i \eta_{11}^{d} S_{3}^{2}-2 i \eta_{13}^{d} S_{1} S_{3}+i \eta_{33}^{d} S_{1}^{2}, \\
\varepsilon_{12}=-i\left(\eta_{23}^{d}+\gamma_{11} S_{1}+\gamma_{13} S_{3}\right) S_{1}+i\left(\eta_{12}^{d}-\gamma_{13} S_{1}-\gamma_{33} S_{3}\right) S_{3}, \\
\varepsilon_{21}=-i\left(\eta_{23}^{d}-\gamma_{11} S_{1}-\gamma_{13} S_{3}\right) S_{1}+i\left(\eta_{12}^{d}+\gamma_{13} S_{1}+\gamma_{33} S_{3}\right) S_{3} .
\end{gathered}
$$


By using formulae (14), we get

$$
\begin{aligned}
& \mu=i\left(\eta_{22}^{d}+\eta_{11}^{d} S_{3}^{2}-2 \eta_{13}^{d} S_{1} S_{3}+\eta_{33}^{d} S_{1}^{2}\right) / 2, \\
& \xi=i\left(\eta_{22}^{d}-\eta_{11}^{d} S_{3}^{2}+2 \eta_{13}^{d} S_{1} S_{3}-\eta_{33}^{d} S_{1}^{2}\right) / 2, \\
& \eta=i\left(\eta_{12}^{d} S_{3}-\eta_{23}^{d} S_{1}\right), \\
& \zeta=-i\left(\gamma_{11} S_{1}^{2}+2 \gamma_{13} S_{1} S_{3}+\gamma_{33} S_{3}^{2}\right) .
\end{aligned}
$$

We see that $\mu, \xi$, and $\eta$ are purely imaginary numbers depending only on dichroic properties of the crystal (absorption). The quantity $\zeta$ depends only on chiral properties of the crystal; $\zeta$ is purely imaginary if the optical activity tensor $\gamma$ is real.

Singularities for crystals with weak dichroism and chirality were studied recently in Berry and Dennis (2003). It was shown that the double coffee filter singularity arises in absorption-dominated crystals, and the sheets of real parts of eigenvalues are separated in chirality-dominated crystals. According to the results of Section 3, these two cases are explicitly determined by the conditions $D>0$ and $D<0$, respectively, where $D=\operatorname{Im}^{2} \xi+\operatorname{Im}^{2} \eta-\operatorname{Im}^{2} \zeta$. These conditions are new and important because they provide quantitative definitions of absorption-dominated and chiralitydominated regimes for unfolding of the diabolic singularity in terms of components of the inverse dielectric tensor. Indeed, according to (61), $\xi$ and $\eta$ depend linearly on all the components of the tensor $\boldsymbol{\eta}_{\text {dichroic }}$, while $\zeta$ depends linearly on the components $\gamma_{i j}, i, j=1,3$ of the optical activity tensor $\gamma$.

Note that according to the sign of the quantity $D$ taken at different optic axes we can classify crystals by their optic properties. For example, the important case is a chirality-dominated crystal with $D<0$ for all four optic axes. Then real parts of the eigenvalues separate for all directions $\mathbf{s}$.

There are four optic axes (55), which determine two pairs of opposite space direction $\pm \mathbf{s}_{0}$. It is easy to see that the unfolding conditions coincide for the optic axes given by opposite directions, while these conditions are different for different pairs of optic axes. In the absorption-dominated case, when diabolic singularities unfold into coffee-filters near two opposite optic axes $\pm \mathbf{s}_{0}= \pm\left(S_{1}, 0, S_{3}\right)$, the four exceptional points of eigenvalue coupling $\pm \mathbf{s}_{a}$ and $\pm \mathbf{s}_{b}$ (also called singular axes) appear. By using (57) in (13), we obtain the asymptotic formulae

$$
s_{1}^{a, b}=S_{1}+\frac{x_{a, b}}{\left(\eta_{1}-\eta_{3}\right) S_{1}}, \quad s_{2}^{a, b}=\frac{y_{a, b}}{\left(\eta_{3}-\eta_{1}\right) S_{1} S_{3}}, \quad s_{3}^{a, b}=\sqrt{1-\left(s_{1}^{a, b}\right)^{2}-\left(s_{2}^{a, b}\right)^{2}},
$$


for the components of the vectors $\mathbf{s}_{a, b}$, where $x_{a, b}$ and $y_{a, b}$ are found by using expressions (24), (25), and (61). In particular, for non-chiral crystals, we have $\zeta=0$. Then expressions (24), (25) yield $x_{a, b}= \pm \operatorname{Im} \eta=\eta_{12}^{d} S_{3}-\eta_{23}^{d} S_{1}$ and $y_{a, b}=\mp \operatorname{Im} \xi=$ $\left(\eta_{22}^{d}-\eta_{11}^{d} S_{3}^{2}+2 \eta_{13}^{d} S_{1} S_{3}-\eta_{33}^{d} S_{1}^{2}\right) / 2$.

Equation $\operatorname{Im} c=0$ determines a line of singularities in the parameter space $\mathbf{p}=$ $\left(s_{1}, s_{2}\right)$. By using (157), (61) in (13), (17), we find this line in the form

$$
\left(s_{1}-S_{1}\right) S_{1}\left(\eta_{1}-\eta_{3}\right) \operatorname{Im} \xi-s_{2} S_{1} S_{3}\left(\eta_{1}-\eta_{3}\right) \operatorname{Im} \eta-\operatorname{Re} \zeta \operatorname{Im} \zeta=0 .
$$

In the absorption-dominated case, line (63) contains two exceptional points $\mathbf{p}_{a, b}=$ $\left(s_{1}^{a, b}, s_{2}^{a, b}\right)$ corresponding to the singular axes $\mathbf{s}_{a, b}$. A segment between the points $\mathbf{p}_{a}$ and $\mathbf{p}_{b}$ corresponds to the coincidence of real parts of the eigenvalues $\operatorname{Re} \lambda_{+}=\operatorname{Re} \lambda_{-}$, while imaginary parts of the eigenvalues $\operatorname{Im} \lambda_{+}=\operatorname{Im} \lambda_{-}$merge at points of line (63) outside this segment, see Figure $2 \mathrm{~b}$. In the chirality-dominated case, when singular axes do not appear, imaginary parts of the eigenvalues $\operatorname{Im} \lambda_{+}=\operatorname{Im} \lambda_{-}$coincide at points of the whole line (63), see Figure 2]. If the optical activity tensor $\gamma$ is real or purely imaginary, then the line of singularities (63) passes through the diabolic point $\mathbf{p}_{0}$, and position of this line does not depend on $\gamma$.

As a numerical example, let us consider a crystal possessing weak absorption and chirality described by the tensors (51), (52) with

$$
\boldsymbol{\eta}_{\text {dichroic }}=\frac{i}{200}\left(\begin{array}{ccc}
3 & 2 & 0 \\
2 & 3 & 1 \\
0 & 1 & 3
\end{array}\right), \quad \boldsymbol{\gamma}=\frac{1}{200}\left(\begin{array}{ccc}
3 & 1 & 2 \\
1 & 3 & 1 \\
2 & 1 & 3
\end{array}\right) .
$$

A corresponding transparent non-chiral crystal is characterized by $\eta_{1}=0.5, \eta_{2}=0.4$, $\eta_{3}=0.1$, and its eigenvalue surfaces with two optic axes are presented in Figure 6 . By using (64) in (61), we find that the condition $D=\frac{7}{160000}(4 \sqrt{3}-5)>0$ is satisfied for the left optic axis $\mathbf{s}_{0}=(-1 / 2,0, \sqrt{3} / 2)$. Hence, the diabolic singularity bifurcates into a double coffee filter with two exceptional points whose coordinates according to expressions (62) are

$$
\mathbf{p}_{a}=\left(-\frac{1}{2}-\frac{1}{80} \sqrt{-35+28 \sqrt{3}}, 0\right), \quad \mathbf{p}_{b}=\left(-\frac{1}{2}+\frac{1}{80} \sqrt{-35+28 \sqrt{3}}, 0\right) .
$$

Local approximations of the eigenvalue surfaces are given by expressions (18), (19), where

$$
\operatorname{Re} c=\frac{35-28 \sqrt{3}}{160000}+\frac{1}{25}\left(s_{1}+1 / 2\right)^{2}+\frac{3}{100} s_{2}^{2}, \quad \operatorname{Im} c=-\frac{6+\sqrt{3}}{2000} s_{2} .
$$



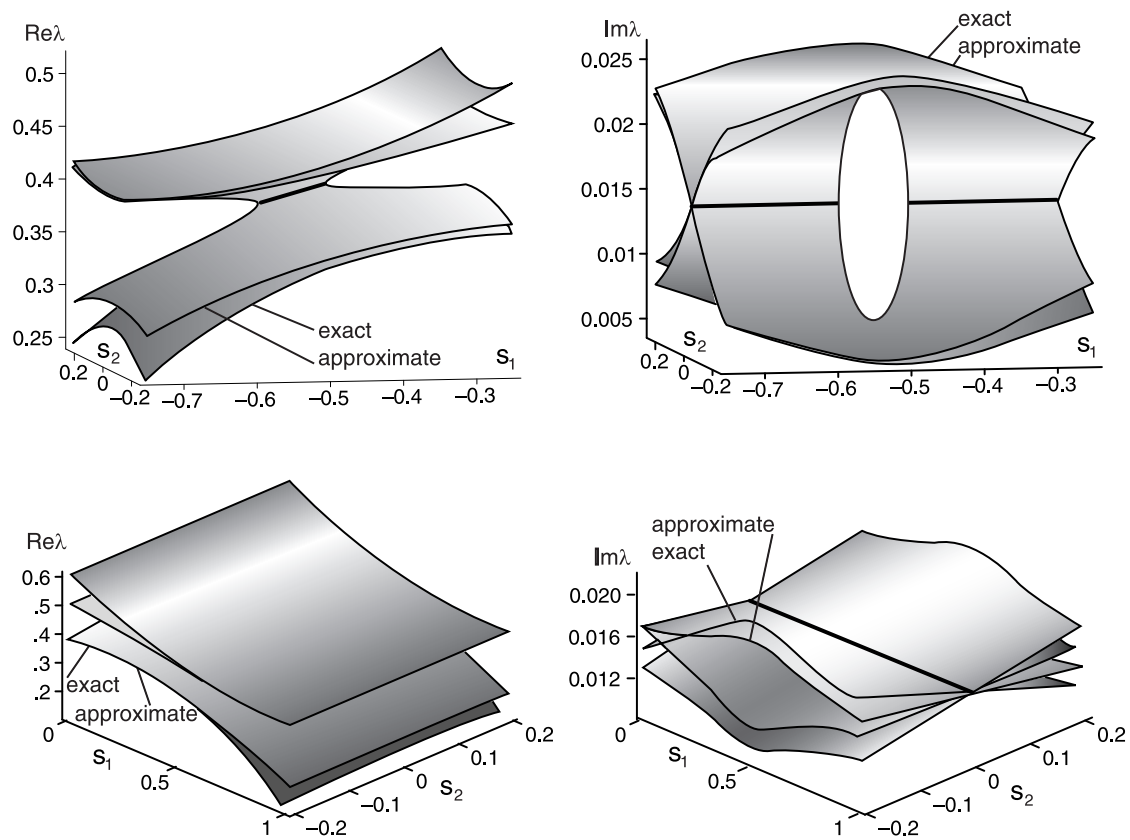

Figure 7: Unfolding of singularities near optic axes.

Figure 7/ shows these local approximations compared with the exact eigenvalue surfaces given by (54). For the right optic axis $\mathbf{s}_{0}=(1 / 2,0, \sqrt{3} / 2)$, the condition $D=-\frac{7}{160000}(4 \sqrt{3}+5)<0$ is satisfied. Hence, the eigenvalue sheets (for real parts) separate under the bifurcation of the right diabolic singularity. Approximate and exact eigenvalue surfaces are shown in Figure $7 \mathrm{~b}$. The approximations are given by expressions (18), (19), where

$$
\operatorname{Re} c=\frac{35+28 \sqrt{3}}{160000}+\frac{1}{25}\left(s_{1}-1 / 2\right)^{2}+\frac{3}{100} s_{2}^{2}, \quad \operatorname{Im} c=-\frac{6-\sqrt{3}}{2000} s_{2} .
$$

We observe that the unfolding types are different for different optic axes. As it is seen from Figure [7, the asymptotic formulae provide an accurate description for unfolding of eigenvalue surfaces near diabolic points.

\section{Conclusion}

Non-Hermitian Hamiltonians and matrices usually appear in physics when dissipative and other non-conservative effects are taken into account. The known examples are complex refractive indices in optics and complex potentials describing the scattering 
of electrons or X-rays. Traditionally, non-Hermitian matrices appear in physics as a perturbation of Hermitian matrices. As it is stated in Berry (2004), Hermitian physics differs radically from non-Hermitian physics in case of coalescence (coupling) of eigenvalues. In the present paper we have studied this important case carefully. We gave analytical description for unfolding of eigenvalue surfaces due to an arbitrary complex perturbation with the singularities known in the literature as a "double coffeefilter" and a "diabolic circle". We emphasize that the developed theory requires only eigenvectors and derivatives of the matrices taken at the singular point, while the size of the matrix and its dependence on parameters are arbitrary. This makes the presented theory powerful and practical for a wide class of physical problems. The given physical example from crystal optics demonstrates applicability and accuracy of the theory.

\section{Acknowledgement}

The work is supported by the research grants RFBR-NSFC 02-01-39004, RFBR 0301-00161, and CRDF-BRHE Y1-MP-06-19.

\section{References}

[Von Neumann and Wigner (1929)] Von Neumann J. and Wigner E.P. Über das Verhalten von Eigenwerten bei adiabatischen Prozessen. Zeitschrift für Physik. 30. 1929. P. 467-470.

[Teller (1937)] Teller E. The Crossing of Potential Surfaces. Journal of Physical Chemistry. 41(1). 1937. P. 109-116.

[Ramachandran and Ramaseshan (1961)] Ramachandran G. N. and Ramaseshan S. Crystal optics. In Handbuch der Physik, vol. XXV/I (ed. H. Flügge). Springer. 1961.

[Berry and Wilkinson (1984)] Berry M.V., Wilkinson M. Diabolical points in the spectra of triangles. Proc. R. Soc. Lond. A. 392. 1984. P. 15-43. 
[Mondragon and Hernandez (1993)] Mondragon A., Hernandez E. Degeneracy and crossing of resonance energy surfaces. J. Phys. A. Math. Gen. 26. 1993. P. 55955611.

[Mondragon and Hernandez (1996)] Mondragon A., Hernandez E. Berry phase of a resonant state. J. Phys. A. Math. Gen. 29. 1996. P. 2567-2585.

[Shuvalov and Scott (2000)] Shuvalov A.L. and Scott N.H. On singular features of acoustic wave propagation in weakly anisotropic thermoviscoelasticity. Acta Mechanica. 140. 2000. P. 1-15.

[Dembowsky et al. (2001)] Dembowsky C., Gräf H.D., Harney H.L., Heine A., Heiss W.D., Rehfeld H., and Richter A. Experimental observation of the topological structure of exceptional points. Phys. Rev. Lett. 86(5). 2001. P. 787-790.

[Dembowsky et al. (2003)] Dembowsky C., Dietz B., Gräf H.D., Harney H.L., Heine A., Heiss W.D., and Richter A. Observation of a Chiral State in a Microwave Cavity, Phys. Rev. Lett. 90. 2003. 034101.

[Keck et al. (2003)] Keck F., Korsch H.J., Mossmann S. Unfolding a diabolic point: a generalized crossing scenario. J. Phys. A: Math. Gen. 36. 2003. P. 2125-2137.

[Korsch and Mossman (2003)] Korsch H.J., Mossmann S. Stark resonances for a double $\delta$ quantum well: crossing scenarios, exceptional points and geometric phases. J. Phys. A. 36. 2003. P. 2139-2153.

[Berry and Dennis (2003)] Berry M.V. and Dennis M.R. The optical singularities of birefringent dichroic chiral crystals. Proc. R. Soc. Lond. A. 459. 2003. P. 12611292.

[Stehmann et al. (2004)] Stehmann T., Heiss W. D. and Scholtz F. G. Observation of exceptional points in electronic circuits, J. Phys. A. Math. Gen. 37. 2004. P. $7813-7819$

[Heiss (2004)] Heiss W. D. Exceptional points of non-Hermitian operators, J. Phys. A. Math. Gen. 37. 2004. P. 2455-2464. 
[Berry (2004)] Berry M.V. Physics of non-Hermitian degeneracies. Czech. J. Phys. 2004.

[Mailybaev et al. (2004)] Mailybaev A.A., Kirillov O.N., and Seyranian A.P. Coupling of eigenvalues of complex matrices at diabolic and exceptional points J. Phys. A. Math. Gen. (submitted).

[Arnold (1972)] Arnold V.I. Modes and quasimodes. Funkcional. Anal. i Prilozhen. 6(2). 1972. P. 12-20. (in Russian).

[Landau et al. (1984)] Landau L.D., Lifshitz E.M., Pitaevskii L.P. Electrodynamics of continuous media. Pergamon: Oxford. 1984.

[Lewin (1994)] Lewin M. On the coefficients of the characteristic polynomial of a matrix. Discrete Mathematics. 125(1-3). 1994. P. 255-262. 